Pacific Journal of Mathematic 


\title{
ASYMPTOTIC PRIME DIVISORS AND GOING DOWN
}

\author{
Stephen McAdam
}

Let $I$ be an ideal in a commutative Noetherian domain $R$, and let $\hat{I}$ be the integral closure of $I$. It is known that the sequences of sets of primes $A s s\left(R / I^{n}\right)$ and $A s s\left(R / \hat{I}^{n}\right)$ both eventually become constants, those constants denoted $A^{*}(I)$ and $\hat{A}^{*}(I)$ respectively. The main result of this paper is that if $T$ is an integral extension of a local domain $(R, M)$, and if $I$ is an ideal of $R$ such that $T / I T$ contains a height 0 maximal ideal, then $M \in \hat{A}^{*}(I)$. This fact is then used to study when $\hat{A}^{*}(P)=\{P\}$ for $P$ a prime of $R$. (This is a variation of the question when does $P^{n}=P^{(n)}$ for all large $n$ ?) It is shown that if $\hat{A}^{*}(P)=\{P\}$, then "going down to $P$ " holds. Finally, the main argument is used to produce an example of an $n$ dimensional local domain, $(R, M)$ such that for any $P \in \operatorname{Spec} R$ $-\{0\}$, and any $m \geq 2, M \in \operatorname{Ass}\left(R / P^{m}\right)$. Also the analytic spread of any such $P$ is $n$.

Background. The question concerning the asymptotic behavior of $\operatorname{Ass}\left(R / I^{n}\right)$ and $\operatorname{Ass}\left(R / \hat{I}^{n}\right)$ was posed in [12] which essentially showed the existence of $\hat{A}^{*}(I)$ (also see [9, Proposition 7]). The existence of $A^{*}(I)$ was proved in [1].

Notation. Throughout, $R$ will be a Noetherian domain with integral closure $R^{\prime}$. If $I$ is an ideal of $R, \hat{I}$ will be the integral closure of $I$. If $R$ is local, $v(I)$ will be the minimal number of generators of $I$ and $l(I)$ will be the analytic spread of $I$. Finally, " $\subset$ " will denote proper containment.

\section{The Main Argument.}

Theorem 1.1. Let $I$ be an ideal in a local domain $(R, M)$. Let $T$ be an integral extension domain of $R$ and assume that T/IT contains a height 0 maximal ideal. Then there is an integer $n \geqq 1$ with the following property: If $J$ is any ideal of $R$ satisfying $I^{m} \subseteq J \subseteq \widehat{M}^{n}$ (any $\left.m \geqq 1\right)$, then $M \in \operatorname{Ass}(R / J)$.

Proof. We first reduce to the case that $T$ is a finite $R$-module contained in $R^{\prime}$. By a simple going up argument, $T^{\prime} / I T^{\prime}$ contains a height 0 maximal ideal. Since $R^{\prime} \subseteq T^{\prime}$ satisfies going down, $R^{\prime} / I R^{\prime}$ has a height 0 maximal, say $N^{\prime} / I R^{\prime}$. Choose $u \in N^{\prime}$ but $u$ in no other maximal ideal of $R^{\prime}$. Let $N=N^{\prime} \cap R[u]$. Then $N^{\prime}$ is the only prime of $R^{\prime}$ lying over $N$. We now easily see that $N / I R[u]$ is 
a height 0 maximal of $R[u] / I R[u]$. Thus we may assume that $T=R[u]$ is a finite $R$-module contained in $R^{\prime}$.

We have a maximal ideal $N$ of $T$ with $N$ minimal over $I T$. Let $(V, P)$ be a D.V.R. overring of $T$ with $P \cap T=N$. Choose $b \in R$ with $b T \subseteq R$, and select $n$ sufficiently large that $b \notin P^{n}$. Since $N$ is minimal over $I^{m} T$, for some $s \in T-N$ and integer $k \geqq 1$, we have $s N^{k} \subseteq I^{m} T$. Thus $b s M^{k} \subseteq b s N^{k} \subseteq I^{m}(b T) \subseteq I^{m} R=I^{m}$. We also claim that $b s \notin \hat{M}^{n}$, since if $b s \in \hat{M}^{n} \cong \hat{N}^{n} \cong \hat{P}^{n}=P^{n}$, since $s$ is a unit of $V$ we have $b \in P^{n}$, a contradiction. Now if $I^{m} \cong J \subseteq \widehat{M}^{n}$ then clearly $b s M^{k} \subseteq J$ and $b s \in R-J$, showing that $M^{k}$ consists of zero divisors modudo $J$. Thus $M \in \operatorname{Ass}(R / J)$.

REMARK. Our choice of $n$ actually only depended on $b$ and $P$ (that is, on $b$ and $N$ ). In particular this $n$ will work for any ideal $I$ such that $N$ is minimal over $I T$.

Corollary 1.2. Let $I$ be an ideal in a local domain $(R, M)$. If there exists an integral extension domain $T$ of $R$ such that $T / I T$ contains a height 0 maximal ideal, then $M \in \hat{A}^{*}(J)$ for any ideal $J$ of $R$ satisfying $I \subseteq \operatorname{Rad}(J)$.

Proof. With $n$ as in Theorem 1.1, clearly for all $m \geqq n$ we have $I^{m} \cong \widehat{I}^{m} \subseteq \widehat{M}^{n}$. Thus $M \in \operatorname{Ass}\left(R / \hat{I}^{m}\right)$, so that $M \in \hat{A}^{*}(I)$. Since $I \subseteq$ $\operatorname{Rad}(J)$, clearly $T / J T$ contains a height 0 maximal and so similarly we get $M \in \widehat{A}^{*}(J)$.

The converse of Corollary 1.2 if false. That is, there is a local domain $(R, M)$ containing an ideal $I$, such that $M \in \widehat{A}^{*}(J)$ for any ideal $J$ whose radical contains $I$, but such that $R$ is integrally closed and $\operatorname{dim} R / I>0$ (so that no $T$ is in Corollary 1.2 exists, using going down). For this, suppose that $(R, M)$ is a 2-dimensional integrally closed local domain, and that $I=P$ is a height 1 prime ideal with the property that $P$ is not the radical of any principal ideal. Let $P \subseteq \operatorname{Rad}(J)$. If $P=\operatorname{Rad}(J)$, then $J$ is not principal and so by [9, Proposition 21] and [7, Theorem 6] we have $M \in A^{*}(J)=\hat{A}^{*}(J)$. If instead $P \neq \operatorname{Rad}(J)$, then clearly $M=\operatorname{Rad}(J)$ and $M \in \hat{A}^{*}(J)$. As $\operatorname{dim} R / P=1$ and $R$ is integrally closed, the converse of Corollary 1.2 fails. There are domains $(R, M)$ with a prime $P$ as just described. A. Sathaye has shown that $K[X, Y, Z] /\left(Z Y^{2}-X^{3}-Z^{3}\right)$ is such a domain, and [4] constructs a 2-dimensional integrally closed local domain in which no height 1 prime is the radical of a principal ideal.

Ratliff has proved the following lovely pair of theorems. Here $R^{*}$ denotes the completion of the local domain $R$. 
THEOREM A ([13, Theorem 9]). The following are equivalent for a local domain $(R, M)$.

(1) $R^{*}$ contains a depth 1 yrime divisor of 0 .

(2) There is an integer $n \geqq 1$ such that for any ideal $I \subseteq M^{n}$, $M \in \operatorname{Ass}(R / I)$.

THEOREM B ([14, Theorem 1]). The following are equivalent for a local domain $(R, M)$.

(1) $R^{*}$ contains a depth 1 minimal prime.

(2) $R^{\prime}$ contains a height 1 maximal prime.

(3) There is an integer $n \geqq 1$ such that for any ideal $I \subseteq M^{n}$, $M \in \operatorname{Ass}(R / \hat{I})$.

(Note: In Theorem B, Ratliff actually only assumes $\operatorname{Rad} R=0$.)

The equivalence of (1) and (2) in Theorem B follows from an earlier theorem of Ratliff [11, Proposition 3.5]. Ratliff's proof of Theorem B argues that (1) is equivalent to (3). We will now give a more elementary proof that (2) is equivalent to (3). (Note: Condition (iii) below is new.)

Proposition 1.3 ([14, Theorem 1$])$. Let $(R, M)$ be a local domain with integral closure $R^{\prime}$ and completion $R^{*}$. The following are equivalent.

(i) $R^{*}$ contains a depth 1 minimal prime.

(ii) $R^{\prime}$ contains a height 1 maximal prime.

(iii) There is an $n \geqq 1$ such that for any ideal $I \subseteq \widehat{M}^{n}, M \in$ $\operatorname{Ass}(R / I)$.

(iv) There is an $n \geqq 1$ such that for any ideal $I \cong M^{n}, M \in$ $\operatorname{Ass}(R / \hat{I})$.

(v) For any ideal $I$ of $R$, there is an $n \geqq 1$ with $M \in \operatorname{Ass}\left(R / \hat{I}^{n}\right)$.

(vi) $M \in \operatorname{Ass}(R / \hat{a})$ for some $a \in R$.

Proof. (i) $\Leftrightarrow$ (ii): By [11, Proposition 3.5]:

(ii) $\Rightarrow$ (iii): Let $N^{\prime}$ be a height 1 maximal of $R^{\prime}$. If $u \in N^{\prime}$ but $\mathrm{u}$ is not in any other maximal prime of $R^{\prime}$, then let $T=R[u]$ and $N=N^{\prime} \cap T$, so that height $N=1$. For any ideal $I$ of $R, N$ is minimal over IT. By the remark following the proof of Theorem 1.1, we see that (iii) is satisfied by the $n$ constructed in Theorem 1.1.

(iii) $\Rightarrow$ (iv): If $I \subseteq M^{n}$ then $\hat{I} \subseteq \hat{M}^{n}$.

(iv) $\Rightarrow(\mathrm{v})$ : Straightforward.

(v) $\Rightarrow$ (vi): Straightforward.

(vi) $\Rightarrow$ (ii): Since $\widehat{a R}=a R^{\prime} \cap R, M$ can be lifted to a prime divisor of $a R^{\prime}$ in $R^{\prime}$. This prime will have height 1 . 
REMARK. Theorem A together with [13, Remark 12.1] show that in conditions (iv), (v), and (vi) above we must use the integral closures of the ideals.

Going down and $\hat{A}^{*}(P)$. A question which has received some attention is when does $P^{n}=P^{(n)}$ for $P$ a prime ideal. For this to hold for all large $n$ is obviously equivalent to $A^{*}(P)=\{P\}$. We pose the question, when does $\hat{A}^{*}(P)=\{P\}$ ? (Since $\hat{A}^{*}$ appears to be better behaved than $A^{*}$, as evidenced by [7, Theorem 3] for example, our version of the question might be more tractable than the other version.) In this section, we will show that $\hat{A}^{*}(P)=\{P\}$ implies a pleasant going down property. We will then show that that going down property often fails.

DEFINITION. Let $P \subset Q$ be primes in a domain $R$. We will say that $P \subset Q$ satisfies going down if for any integral extension domain $T$ of $R$ and any prime $q$ of $T$ with $q \cap R=Q$, there is a prime $p$ of $T$ with $p \subset q$ and $p \cap R=P$.

Proposition 2.1. Let $P \subset Q$ be primes in a Noetherian domain $R$. If $P \subset Q$ does not satisfy going down, then there is a prime $Q_{1}$ of $R$ with $P \subset Q_{1} \leqq Q, P \subset Q_{1}$ does not satisfy going down, and $Q_{1} \in$ $\hat{A}^{*}(P)$.

Proof. Let $T$ be an integral extension domain of $R$ and let $q$ be prime in $T$ with $q \cap R=Q$ such that there is no prime $p$ of with $p \subset q$ and $p \cap R=P$. Choose $q_{1}$ prime in $T$ with $P T \leqq q_{1} \leqq q$ and $q_{1}$ minimal over $P T$. Let $Q_{1}=q_{1} \cap R$. Clearly $P \subset Q_{1}$ fails going down. Letting $S=R-Q_{1}$ and considering $R_{S} \subset T_{S}$, since $\left(q_{1}\right)_{S}$ is minimal over $P_{S} T_{S}$, by Corollary 1.2 we have $\left(Q_{1}\right)_{S} \in \hat{A}^{*}\left(P_{S}\right)$. Thus $Q_{1} \in \hat{A}^{*}(P)$.

Corollary 2.2. Let $P$ be prime in a Noetherian domain $R$. If $\hat{A}^{*}(P)=\{P\}$, then $P \subset Q$ satisfies going down for any prime $Q$ containing $P$.

Proof. Immediate.

REMARK. The converse of Corollary 2.2 fails. Let $(R, M)$ be a 2-dimensional integrally closed local domain, and let $P$ be a nonprincipal height 1 prime of $R$. Since $R$ is integrally closed, $P \subset M$ satisfies going down. However, by [9, Proposition 21] and [7, Theorem 6], $M \in A^{*}(P)=\hat{A}^{*}(P)$. (Notice that such an $(R, M)$ is Macaulay, and hence satisfies the altitude formula.) 
CoROllary 2.3. Let $P \subset Q$ be primes in a Noetherian domain $R$ with height $(Q / P)=2$. If $P \subset Q$ fails to satisfy going down, then for all but finitely many primes $p$ with $P \subset p \subset Q$, we have $Q \in \widehat{A}^{*}(p)$. For those $p$ with $Q \notin \hat{A}^{*}(p)$ we have $p \in \hat{A}^{*}(P)$.

Proof. Suppose $P \subset p \subset Q, p$ prime. If $p \notin \hat{A}^{*}(P)$ then Proposition 2.1 easily shows that $P \subset p$ satisfies going down. Since $P \subset Q$ fails going down, obviously $p \subset Q$ must fail going down. Now by Proposition 2.1, we see that $Q \in \hat{A}^{*}(p)$ for all $p \notin \hat{A}^{*}(P)$. As $\hat{A}^{*}(P)$ is finite, we are done.

It is not difficult to produce situations in which $P \subset Q$ fails to satisfy going down, as is illustrated by [5, Theorem 2] or [6]. As an example of how the arguments in [5] or [6] can be combined with the present arguments, we present the following.

Proposition 2.4. Let $R$ be a Noetherian domain with integral closure $R^{\prime}$. Let $Q$ be a prime of $R$ with height $Q \geqq 2$. Suppose that in $R^{\prime}$, more than one prime lies over $Q$. Then there are infinitely many primes $P$ of $R$ satisfying $P \subset Q$, height $(Q / P)=1$ and $Q \in \hat{A}^{*}(P)$.

Proof. A simple variation of the proof of [5, Theorem 2] shows that there are infinitely many primes $P \subset Q$ with $P \subset Q$ failing to satisfy going down. If infinitely many such $P$ also satisfy height $(Q / P)=1$ then for each of these we have $Q \in \hat{A}^{*}(P)$ by Proposition 2.1, and we are done. Otherwise for some $P \subset Q$ which fails going down, wehave height $(Q / P)>1$. We claim that such $P$ can be found with height $(Q / P)=2$. Since infinitely many primes $p$ satisfy $P \subset$ $p \subset Q$ and height $(Q / p)=$ height $(Q / P)-1$, we may pick such a $p$ not in $\hat{A}^{*}(P)$. By Proposition 2.1, (since height $(p / P)=1$ ) we have $P \subset p$ satisfies going down. Thus $p \subset Q$ must fail going down. Our claim now follows by induction. We now have $P \subset Q$, heigh $(Q / P)=2$ and $P \subset Q$ fails going down. The result follows from Corollary 2.3.

We close this section with an observation concerning local domains which satisfy the altitude formula. Recall that $l(I)$ is the analytic spread of the ideal $I$.

Proposition 2.5. Let $(R, M)$ be a local domain which satisfies the altitude formula. Let $P$ be a prime ideal of $R$. Consider the statements (a) $l(P)=$ height $P$; (b) $\widehat{A}^{*}(P)=\{P\}$. Then (a) implies (b) but not conversely.

Proof. Suppose that $P \neq Q \in \hat{A}^{*}(P)$. By [7, Theorem 3], $l\left(P_{Q}\right)=$ 
height $Q>$ height $P$. However it is not difficult to see that $l(P) \geqq$ $l\left(P_{Q}\right)$. Thus (a) fails. Therefore (a) implies (b).

To see that (b) does not imply (a), we consider a 3-dimensional integrally closed local domain $(R, M)$, satisfying the altitude formula and having $R / M$ infinite, with a height 1 prime $P$ whose minimal number of generators $v(P)=2$, but such that $v\left(P_{Q}\right)=1$ for all height 2 primes $Q$. We first see that (b) holds. Since $v\left(P_{Q}\right)=1$, clearly $l\left(P_{Q}\right)=1<$ height $Q=2$. By [7, Theorem 3], $Q \notin \hat{A}^{*}(P)$ for any height 2 prime $Q$. Also $l(P) \leqq v(P)=2<$ height $M=3$. Thus $M \notin \hat{A}^{*}(P)$. Therefore $\hat{A}^{*}(P)=\{P\}$ and (b) holds. To show (a) fails, we need $l(P) \neq$ height $P=1$. If $l(P)=1$ then (since $R / M$ is infinite) there is a principal reduction, $c R$, of $P$. Thus $c R \cong P \cong c \widehat{c}$. As $R$ is integrally closed, $c R=\widehat{c R}$, contradicting $v(P)=2$.

It remains to be seen that such an $(R, M)$ and $P$ exists. $P$. Eakin provided the following example. With $K$ an infinite field and $X, Y, Z, W$ indeterminates, let $R=K[X, Y, Z, W]_{(X, Y, Z, W)} /(X Y-$ $Z W)_{(X, Y, Z, W)}$. This is integrally closed using [15, Theorem 1]. Let $P$ be the image of $(X, Z)$. Since $\bar{X}=\bar{Z}(\bar{W} / \bar{Y})$ and $\bar{Z}=\bar{X}(\bar{Y} / \bar{W})$ and since any height 2 prime $Q$ containing $P$ fails to contain one of $\bar{W}$ or $\bar{Y}, v\left(P_{Q}\right)=1$. Since $(X Y-Z W) \subset(X, Y, Z, W)^{2}$, the maximal ideal of $R$ requires 4 generators. Thus $P$ must not be principal, so $v(P)=2$.

REMARK. We now have that if $P$ is prime in a local domain satisfying the altitude formula, then (i) $\Rightarrow$ (ii) $\Rightarrow$ (iii) and none of the reverse, with

(i) $l(P)=$ height $P$

(ii) $\hat{A}^{*}(P)=\{P\}$

(iii) $P \subset Q$ satisfies going down for any prime $Q$ containing $P$.

We mention that the proof in (i) $\Rightarrow$ (iii) is not difficult. Thus the significance of the above is that (ii) lies properly between (i) and (iii).

An Example. Let $(R, M)$ be a local domain with completion $R^{*}$. In $[13,10.2]$ Ratliff asks if the following two conditions are equivalent.

(I) $R^{*}$ contains a depth 1 prime divisor of 0 .

(II) For $P \in \operatorname{Spec} R-\{0, M\}, P^{n} \neq P^{(n)}$ for all large $n$. That is, $A^{*}(P) \neq\{P\}$.

$((\mathrm{I}) \Rightarrow$ (II) is immediate from Theorem A.) The following example shows that (II) does not imply (I), as well as having other interesting characteristics. 
Example. Let $n \geqq 2$ be an integer. By [3, Theorem A] it is possible to construct a Noetherian domain $T$ with exactly two maximal ideals $N_{1}$ and $N_{2}$ with height $N_{1}=$ height $N_{2}=n$, such that there is a field $F$ and indeterminates $X_{\imath 1}, \cdots, X_{i n} i=1,2$ with $T_{N_{i}}=F\left[X_{\imath 1}, \cdots, X_{\imath n}\right]_{\left(X_{i 1}, \cdots, X_{i n}\right)}$. Thus $T / N_{\imath} \approx F, i=1,2$. Also, for each $0 \neq Q \in \operatorname{Spec} T, Q$ is in exactly one of $N_{1}$ or $N_{2}$. Now let $\varphi$ be a field isomorphism from $T / N_{1}$ onto $T / N_{2}$ and let $R=\{t \in$ $\left.T \mid \varphi\left(t+N_{1}\right)=t+N_{2}\right\}$. By [2, Theorem A], $R$ is a local domain with maximal ideal $M=N_{1} \cap N_{2}$. Since $M T \subseteq R, T$ is a finite $R$-module in the quotient field of $R$, and for each $P \in \operatorname{Spec} R-\{M\}$, there is a unique prime of $T$ lying over $P$.

(a): $R$ is analytically unramified. By $[10, \S 36], T_{N_{i}}$ is analytically unramified $i=1,2$. Thus $T$ is analytically unramified and since $T$ is a finite $R$-module, $R$ is analytically unramified.

(b): $R$ satisfies the altitude formula. For this, Ratliff has shown that it is equivalent to see that $R[X]_{(M, X)}$ is catenary [8, Corollary 2.5]. However this follows easily from the fact that $T[X]_{\left(N_{2}, X\right)}$ is $n+1$-dimensional and catenary, $i=1,2$.

(c): In the completion, $R^{*}$, each prime divisor of 0 is minimal and has depth $n$. Since $R$ is analytically unramified, $\operatorname{Rad} R^{*}=0$ and so each prime divisor of 0 is minimal. Since $R$ satisfies the altitude formula, $R$ is quasi-unmixed so that each minimal prime in $R^{*}$ has depth $n$ [11, Theorem 3.1].

(d): For $P \in \operatorname{Spec} R-\{0, M\}, M \in \hat{A}^{*}(P) \subseteq A^{*}(P)$. Let $Q$ be the unique prime of $T$ lying over $P$. Without loss, we may assume $Q \subset N_{1}$ so that $Q \nsubseteq N_{2}$. We claim that $N_{2}$ is minimal over $P T$. If $P T \subseteq q \subset N_{2}$ with $q$ prime in $T$, let $p=q \cap R$. Since $P \subseteq p$, by going up, $Q$ can be enlarged to a prime lying over $p$. However $q$ is the unique prime of $T$ lying over $p$. Thus $Q \subseteq q \subset N_{2}$. This contradiction proves our claim that $N_{2}$ is minimal over $P T$. By Corollary $1.2, M \in \hat{A}^{*}(P)$.

(e): For $0 \neq P \in \operatorname{Spec} R, l(P)=n$. This follows from (b), (d) and [7, Theorem 3].

(f): If $P \in \operatorname{Spec} R-\{0, M\}$ we in fact have $M \in \operatorname{Ass}\left(R / P^{m}\right)$ for all $m \geqq 2$. Pick $b_{1} \in N_{1}-\left(N_{1}^{2} \cup N_{2}\right)$ and $b_{2} \in N_{2}-\left(N_{2}^{2} \cup N_{1}\right)$. Then $b=$ $b_{1} b_{2} \in\left(N_{1} \cap N_{2}\right)-\left(N_{1}^{2} \cup N_{2}^{2}\right)$. As was argued in (d), we have (say) $N_{2}$ minimal over $P T$. Thus for some $s \in T-N_{2}$ and integer $k \geqq 1$, $s N_{2}^{k} \subseteq P^{m} T$. Therefore $s b M^{k} \subseteq s b N_{2}^{k} \subseteq P^{m}(b T) \leqq P^{m} R=P^{m} \quad$ (since $b \in N_{1} \cap N_{2}=M$ and $M T \subseteq R$ ). Now $s b \in R-P^{m}$ since if $s b \in P^{m} \subseteq$ $M^{m} \subseteq M^{2} \subseteq N_{2}^{2}$, then since $s \in T-N_{2}$ we would have $b \in N_{2}^{2}$, a contradiction. As $s b M^{k} \leqq P^{m}$ and $s b \in R-P^{m}, M^{k}$ consists of zero divisors modulo $P^{m}$ so that $M \in \operatorname{Ass}\left(R / P^{m}\right)$ for any $m \geqq 2$.

REMARK. In fact (II) $\Rightarrow$ (I) in Ratliff's question, even if $R$ is 
integrally closed. [4] constructs a 2-dimensional integrally closed local domain $(R, M)$ in which each height 1 prime is nonprincipal. By [9, Proposition 21] $R$ satisfies (II). Also 2-dimensional integrally closed implies $R$ is Macaulay so that (I) is known to fail.

\section{REFERENCES}

1. M. Brodmann, Asymptotic stability of Ass (R/I $\left.{ }^{n}\right)$, P.A.M.S., (to appear).

2. A. M. Doering and Y. Lequain, The glueing of maximal ideals, (manuscript).

3. R. Heitmann, Prime ideal posets in Noetherian rings, Rocky Mountain J. Math., 7 (1977),. 667-673.

4. H. B. Laufer, On generalized Weierstrass points and rings with no radical principal prime ideals, (manuscript).

5. S. McAdam, Going down, Duke J. Math., 39 (1972), 633-636.

6. - 1-going down, J. Landon Math. Soc., 2 (1974), 674-680.

7. - Asymptotic prime divisors and analytic spreads, P.A.M.S., (to appear).

8. S. McAdam and E. Davis, Prime divisors and saturated chains, Indiana Univ. Math.

J., 26 (1977), 653-662.

9. S. McAdam and P. Eakin, The asymptotic ass., J. Algebra, 61 (1979), 71-81.

10. M. Nagata, Local Rings, Intersciences, New York, 1962.

11. L. J. Ratliff, Jr., On quasi-unmixed local domains, the altitude formula, and the chain condition for prime ideals (I), Amer. J. Math., X (1969), 502-528.

12. - On prime divisors of $I^{n}, n$ large, Michigan Math. J., 23 (1976), 337-352.

13. Two theorems on the prime divisors of zero in completions of local domains, Pacific J. Math., (to appear).

14. - Notes on integrally closed ideals and asymptotic prime divisors, (manuscript).

15. J. Sally, A note on integral closure, (manuscript).

Received November 30, 1979.

The University of Texas

Austin, TX 78712 


\section{PACIFIC JOURNAL OF MATHEMATICS}

\section{EDITORS}

DoNALD BABBITT (Managing Editor)

University of Galifornia

Los Angeles, California 90024

HUgo RossI

University of Utah

Salt Lake City, UT 84112

C. C. MOORE AND ANDREW OGG

University of California

Berkeley, CA 94720
J. DugunduI

Department of Mathematics

University of Southern California

Los Angeles, California 90007

R. Finn and J. Milgram

Stanford University

Stanford, California 94305

\section{ASSOCIATE EDITORS}
E. F. BeCKenbaCh
B. H. NeumanN
F. WOLF
K. YoSHIDA

\section{SUPPORTING INSTITUTIONS}

UNIVERSITY OF BRITISH COLUMBIA

CALIFORNIA INSTITUTE OF TECHNOLOGY

UNIVERSITY OF CALIFORNIA

MONTANA STATE UNIVERSITY

UNIVERSITY OF NEVADA, RENO

NEW MEXICO STATE UNIVERSITY

OREGON STATE UNIVERSITY

UNIVERSITY OF OREGON
UNIVERSITY OF SOUTHERN CALIFONIA STANFORD UNIVERSITY UNIVERSITY OF HAWAII UNIVERSITY OF TOKYO UNIVERSITY OF UTAH WASHINGTON STATE UNIVERSITY UNIVERSITY OF WASHINGTON 


\section{Pacific Journal of Mathematics}

\section{Vol. 91, No. $1 \quad$ November, 1980}

Harvey Leslie Abbott, Extremal problems on nonaveraging and nondividing

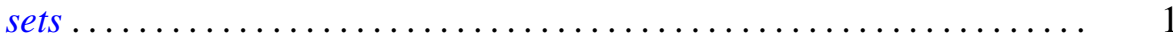

Marine Bruce Abrahamse and Stephen D. Fisher, Mapping intervals to

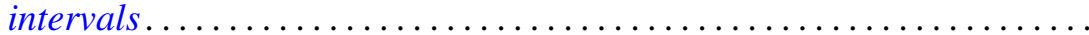

William Wells Adams, The best two-dimensional Diophantine

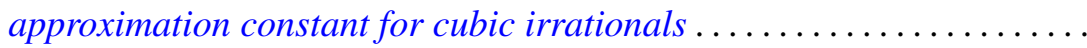

Marilyn Breen, A quantitative version of Krasnosel'skiu 's theorem in

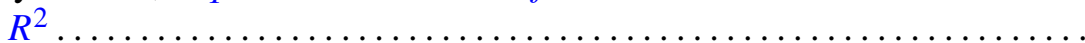

Stephen LaVern Campbell, Linear operators for which $T^{*} T$ and $T T^{*}$

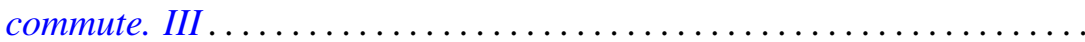

Zvonko Cerin, On cellular decompositions of Hilbert cube manifolds ......

J. R. Choike, Ignacy I. Kotlarski and V. M. Smith, On a characterization

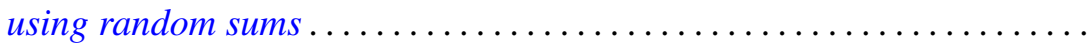

Karl-Theodor Eisele, Direct factorizations of measures .............. 79

Douglas Harris, Every space is a path component space ............. 95

John P. Holmes and Arthur Argyle Sagle, Analytic H-spaces, Campbell-Hausdorff formula, and alternative algebras.............

Richard Howard Hudson and Kenneth S. Williams, Some new residuacity criteria ..........................................

V. Karunakaran and Michael Robert Ziegler, The radius of starlikeness for a class of regular functions defined by an integral ....

Ka-Sing Lau, On the Banach spaces of functions with bounded upper

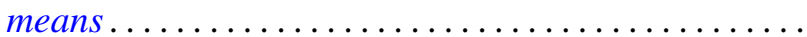

Daniel Paul Maki, On determining regular behavior from the recurrence formula for orthogonal polynomials................

Stephen Joseph McAdam, Asymptotic prime divisors and going down...

Douglas Edward Miller, Borel selectors for separated quotients ..

Kent Morrison, The scheme of finite-dimensional representations of an algebra

Donald P. Story, A characterization of the local Radon-Nikodým property by tensor products

Arne Stray, Two applications of the Schur-Nevanlinna algorithm ...

N. B. Tinberg, The Levi decomposition of a split $(B, N)$-pair ...

Charles Irvin Vinsonhaler and William Jennings Wickless, A theorem on quasi-pure-projective torsion free abelian groups of finite rank... 